\title{
Adiponectin, leptin and TNF- $\alpha$ serum levels in obese and normal weight Peruvian adults with and without chronic periodontitis
}

\author{
Gerardo Mendoza-Azpur ${ }^{1}$, Carmen Castro ${ }^{1}$, Lizet Peña ${ }^{2}$, Maria-Eugenia Guerrero ${ }^{1}$, Manuel De La Rosa ${ }^{3}$, \\ Claudio Mendes ${ }^{4}$, Leandro Chambrone ${ }^{5}$ \\ ${ }^{1}$ Department of Periodontology, School of Dentistry, Cientifica del Sur University, Lima, Peru \\ ${ }^{2}$ Private dental Practice, Lima, Peru \\ ${ }^{3}$ Department of Periodontology, School of Dentistry, University of Monterrey, Monterrey, Mexico \\ ${ }^{4}$ Division of Periodontics, Department of Stomatology, School of Dentistry, University of São Paulo, São Paulo, SP, Brazil \\ ${ }^{5}$ Department of Periodontics, College of Dentistry, The University of Iowa, USA; and Unit of Basic Oral Investigation (UIBO), \\ School of Dentistry, E1 Bosque University, Colombia
}

Correspondence:

Department of Periodontology - School of Dentistry

Cientifica del Sur University

Av. Paseo de la República

$N^{\circ} 5544$ - Lima 18, Peru

mega43@hotmail.com

Received: 03/02/2015

Accepted: 26/03/2015

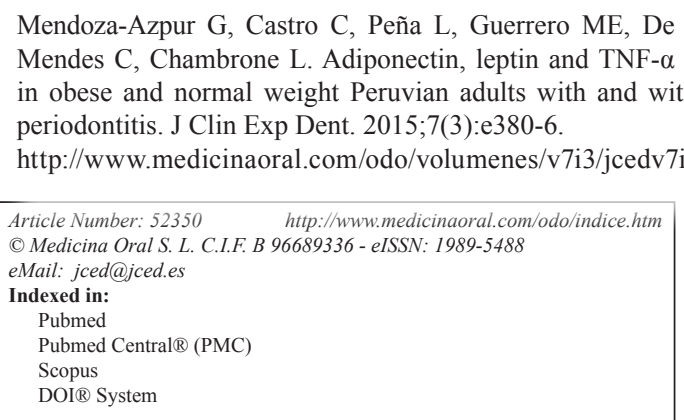

\begin{abstract}
Background: TNF- $\alpha$, an adipokine involved in systemic inflammation and a member of a group of cytokines that stimulate the acute phase reaction, has been related to the pathogenesis of both periodontitis and obesity. The objective of this study was to assess the serum levels of adiponectin, leptin and TNF- $\alpha$ of periodontally healthy normal weight (NW) patients, NW patients with chronic periodontitis (CP), periodontally healthy obese patients and obese patients with CP.

Material and Methods: Ninety-three patients were enrolled in this cross-sectional study: 30 periodontally healthy NW patients; $18 \mathrm{NW}$ patients with CP; 21 periodontally healthy obese patients; and 24 obese patients with CP. Analyses included clinical and anthropometric outcomes, as well as the assessment of serum levels of adiponectin, leptin and TNF- $\alpha$ by enzyme linked immunosorbent assay (ELISA) or fully automated chemiluminescence immunoassay. One-Way ANOVA, Kruskal-Wallis One-Way on Ranks, Dunn's Test and multivariable logistic regression (MLR) analyses were conducted to estimate the degree of association between periodontitis and obesity.

Results: Obese patients with $\mathrm{CP}$ showed significant more bleeding sites than the other three groups $(p<0.05)$. Moreover, patients from the NWCP and OPH showed similar BOP percentages, as well as OPH group showed more bleeding sites than the NWPH group $(p<0.05)$. The OPH group showed similar levels of adiponectin and leptin than the OCP group, but significantly higher than the NWPH and NWCP groups $(p<0.05)$. MLR analyses showed that obesity was positively associated with the percentage of sites with bleeding on probing, with an odds ratio of 0.93 (95\% confidence interval: $-0.88,-0.98 ; p=0.012$ ).

Conclusions: The serum levels of adiponectin, leptin and TNF- $\alpha$ were not influenced by CP. Obese patients showed almost $10 \%$ more sites with BoP. In chronic periodontitis patients, obese subjects presented significant more BOP sites than normal weight subjects.
\end{abstract}

Key words: Periodontitis, obesity, inflammation, adiponectin, leptin. 


\section{Introduction}

It is well-established that the development and progression of chronic periodontitis $(\mathrm{CP})$ is directly linked to dental biofilm accumulation on dental surfaces (1-3). If left undisturbed, dental plaque biofilms stimulate the inflammation of the periodontal tissue, as well as an activation of a ceaseless host response that may trigger periodontal destruction (1). Despite being focal and confined to the oral cavity, CP generates an inflammatory burden that may interact with other body environments, systemic diseases and conditions, such as adverse pregnancy outcomes (4), atherosclerotic cardiovascular disease (5), chronic kidney disease (6) and diabetes mellitus $(7,8)$. The progression of CP may also be affected by other conditions such as smoking (9-11) and diabetes mellitus (7). It has been suggested, that this complex and concomitant interplay of different biologic processes seems to associate periodontitis to obesity (12). Obesity, defined by the World Health Organization (WHO) (13) was abnormal or excessive fat accumulation that may impair health', is evaluated by the body mass index (BMI), i.e. a person's weight in kilograms divided by the square of his height in meters $\left(\mathrm{kg} / \mathrm{m}^{2}\right)$. The WHO estimates that more than 1.4 billion adults $>20$ years of age are overweight, and out of this number, more than of 500 million are obese. Greater than 2.8 million people die every year as a consequence of being overweight and/or obesity. These conditions are also associated to $44 \%$ of the diabetes mellitus burden and $23 \%$ of the population suffering from ischemic heart disease (13).

Efforts have been made to comprehend the endocrine features of white adipose tissue and its role in the production of two important adipocytokines: adinopectin and leptin (14). The first is an anti-inflammatory protein that modulates glucose metabolism, fatty acids, and immune response, whereas the second is a pro-inflammatory peptide hormone that regulates food intake, energy balance, fat storage and the secretion of other cytokines $(14,15)$. Reduced serum concentrations of adiponectin are associated with obesity, insulin resistance and type 2 diabetes mellitus $(14,16)$. Conversely, obese subject may present elevated serum leptin levels (17).

Systematic review outcomes revealed that obese subjects may present more attachment loss than non-obese subjects, and that patients with periodontitis reported greater body mass indexes (BMI) than periodontally healthy subjects $(18,19)$. These studies also suggested that chronic inflammatory processes occurring in the periodontium of patients with periodontal disease may lead to systemic inflammation and secretion of locally produced pro-inflammatory cytokines/mediators $(18,19)$. TNF- $\alpha$, an adipokine involved in systemic inflammation and a member of a group of cytokines that stimulate the acute phase reaction, has been related to the pathogenesis of both periodontitis (20) and obesity (21).
Previous studies have reported increased serum levels of leptin in patients with periodontitis $(17,22)$. More recent investigations (23) reported that periodontitis was associated with reduced levels of serum adiponectin, and that obesity and periodontitis may regulate the serum levels of leptin in favor of proinflammation. In addition, these authors found amongst subjects with periodontitis,obese or normal weighing, a significant increase in TNF- $\alpha$ levels in sites with probing depths greater than $5 \mathrm{~mm}$.

Thus, the objective of this study was to assess the serum levels of adiponectin, leptin and TNF- $\alpha$ of periodontally healthy $(\mathrm{PH})$ normal weight $(\mathrm{NW})$ subjects, NW subjects with chronic periodontitis (CP), $\mathrm{PH}$ obese subjects and obese subjects with CP.

\section{Material and Methods}

-Ethical Consideration and Study Design

This cross-sectional study was approved by the Ethics on Research Committee of the Faculty of Dentistry at the Científica del Sur University, and was conducted in accordance with the Helsinki Declaration of 1975 as revised in the year 2000. The subjects participating in the study were volunteers who received detailed information regarding the proposed research and provided signed informed consent. In addition, the study design was prepared according to The Strengthening the Reporting of Observational Studies in Epidemiology (STROBE) statement (24).

-Study Population and Inclusion Criteria

Ninety three systemically healthy, non-smoking subjects, 25 male and 68 female, 30 to 60 years old (mean age: 42.6 years), participated in the study. These subjects were selected from an initial sample of 600 patients who were referred for dental treatment at the dental clinic of Científica del Sur University and at two private practices between July 2012 and January 2013. The patients were enrolled in the study when they met the following inclusion criteria: 1) age $>30$ years; 2) at least 15 teeth, excluding third molars; 3) non-smoking status; and 4) a normal weight or obese type I or II status (13). All consecutive patients who met these inclusion criteria were invited to participate in the study. Patients with a diagnosis of gingivitis or aggressive periodontitis, a known systemic disease such asacquired immunodeficiency syndrome [AIDS] or diabetes mellitus, pregnant/ lactating, currently undergoing orthodontic treatment or submitted to periodontal therapy within the previous 12 months, or having undergone antimicrobial, anti-inflammatory and/or immunosuppressive therapies during the previous 6 months were excluded from the study.

-BMI Assessment

BMI was calculated based on each subject's weight in kilograms divided by the square of his height in meters $\left(\mathrm{kg} / \mathrm{m}^{2}\right)$. Weight and height measurements were performed by the same trained examiners. Subjects were clas- 
sified as obese if they presented. Obesity was defined according to the WHO (2012) as type I (i.e. BMI greater than or equal to $30 \mathrm{~kg} / \mathrm{m}^{2}$ but less than $35 \mathrm{~kg} / \mathrm{m}^{2}$ ) or type II (i.e. BMI greater than or equal to $35 \mathrm{~kg} / \mathrm{m}^{2}$ but less than $40 \mathrm{~kg} / \mathrm{m}^{2}$ ). NW was defined as BMI ranging from 20 to $24.9 \mathrm{~kg} / \mathrm{m}^{2}$.

-Clinical examination

Full medical and dental histories were obtained. Data included full mouth probing depth (PD) measured at six sites around the teeth, clinical attachment level (CAL) and presence or absence of bleeding on probing using a UNC-15 periodontal probe recorded by two trained and calibrated periodontists. (kappa intra-class correlation coefficient $>0.82$ for PD and CAL). Patients without a history of periodontitis, no sites with $\mathrm{PD}$ and $\mathrm{CAL}>3$ $\mathrm{mm}$ concomitantly were included in the periodontally healthy groups. Those exhibiting bleeding on probing in at least four teeth with a PD $>4 \mathrm{~mm}$ were entered to the chronic periodontitis group. Chronic periodontitis (CP) was defined according to the criteria established by the World Workshop and the American Academy of Periodontology (25).

-Sample Size Calculation and Experimental Groups

Sample size calculation was performed based on the findings of a previous study (23). The primary outcome was considered TNF alpha levels. Considering an expected standard deviation of $2.8 \mathrm{pg} / \mathrm{ml}$, the required number of subjects to detect a relevant mean difference of $2.5 \mathrm{pg} / \mathrm{ml}$ between any two of the four groups (alpha $=5 \%$, power $=$ $80 \%$ ) was estimated at 17 patients for each group.

Following the assessment of clinical and BMI outcomes, subjects were divided into four groups: 1) NWPH $(\mathrm{n}=30)$ : NW subjects without $\mathrm{CP} ; 2) \mathrm{NWCP}(\mathrm{n}=18)$ : NW subjects with $\mathrm{CP} ; 3) \mathrm{OPH}(\mathrm{n}=21)$ : Obese subjects without $\mathrm{CP}$; and 4) OCP $(\mathrm{n}=24)$ : Obese subjects with $\mathrm{CP}$.

-Serum sampling

Serum samples were obtained as described in a previous investigation (23). Peripheral blood samples were collected in the morning and one week after clinical examination into a proper tube. Subsequently, the serum was separated from blood by centrifugation (10 $\mathrm{min}$ at $1,300 \mathrm{rpm}$ ) and stored at $-80^{\circ} \mathrm{C}$. Serum levels of adiponectin and leptin were analyzed by enzyme linked immunosorbent assay (ELISA), as well as TNF- $\alpha$ by fully automated chemiluminescence immunoassay. The ranges of detection for adiponectin, leptin and TNF- $\alpha$ were 2.0 to $20.0 \mathrm{ug} / \mathrm{ml}$ (male) and 4.0 to $22.0 \mathrm{ug} / \mathrm{ml}$ (female), 1.0 to $10.0 \mathrm{ng} / \mathrm{ml}$ (male) and 4.0 to $25.0 \mathrm{ng} / \mathrm{ml}$ (female), and 2.0 to $7.8 \mathrm{pg} / \mathrm{ml}$, respectively. In addition, serum sampling was performed at a biomedical laboratory contracted for such specific purpose, and sample tubes were identified only by the name of the subject.

-Statistical Analysis

Descriptive statistics were used to synthesize collected data. The modified-Levene test was applied to test for equality of variance. Differences between the groups for Age, BoP, CAL, adiponectin, leptin, and TNF- $\alpha$ were analysed using One-Way Analysis of Variance (ANOVA) or Kruskal-Wallis One-Way ANOVA on Ranks (when the variances were considered not equal by Modified Levene Equal-Variance test). Kruskal-Wallis Multiple-Comparison Z-Value Test (Dunn's Test) was applied when significant differences were found.

Two sets of multivariable logistic regression (MLR) models were performed to investigate the association between periodontitis and obesity using each patient as the unit of analysis - a total of six MLR analyses were performed, three testing the influence of $\mathrm{CP}$ on obesity and three testing the influence of obesity on CP. Odds ratio (OR) with a $95 \%$ confidence interval were calculated. The first MLR analysis explored the relationship between age, BOP, gender and $\mathrm{CP}$ and obesity (the dependent variable was the diagnosis of obesity). The second and third analyses assessed the same independent variables versus the specific diagnosis of obesity. Adipocytokines outcomes' were not included in these analyses since obese subjects present significant higher serum levels than normal weighted subjects (13).

The fourth MLR model evaluated the influence of age, gender, obesity and serum levels of adipocytokines with CP (the dependent variable was the diagnosis of CP). For the fifth and sixth MLR analyses, the independent variables were the serum levels of adipocytokines; and the dependent variables were the patients with $\mathrm{CP}+$ obesity (overall analysis) and patients with $\mathrm{CP}+$ obesity presenting sites with $\mathrm{PD}>5 \mathrm{~mm}$, respectively. For these models, BOP and CAL outcomes were not included in these analyses since subjects with $\mathrm{CP}$ present significant BOP and attachment loss than periodontally healthy subjects. The analyses were performed using the NCSS 2007 software package (Number Cruncher Statistical System, NCSS, Kaysville, UT, USA). Differences at $p<$ 0.05 were considered statistically significant.

\section{Results}

Table 1 shows the demographic and periodontal conditions of the four examined groups. There were no significant differences between groups regarding age $(p>0.05)$. For CAL, Kruskal-Wallis Multiple-Comparison Z-Value Test confirmed the similarity between NWPH and OPH groups, as well as between NWCP and OCP groups. Also, differences between periodontally healthy and $\mathrm{CP}$ groups were confirmed $(P<0.05)$. Concerning BOP, obese subjects with $\mathrm{CP}$ showed significant more bleeding sites than the other three groups $(p<0.05)$. Moreover, subjects from the NWCP and OPH showed similar BOP percentages, as well as OPH group showed more bleeding sites than the NWPH group $(p<0.05)$.

With respect to individual adipocytokines outcomes (Table 2), the OPH group showed similar levels of adipo- 
Table 1. Demographic and periodontal characteristics (mean + standard deviations) of the study population.

\begin{tabular}{|l|c|c|c|c|}
\hline Parameters & NWPH & NWCP & OPH & OCP \\
\hline Age (years $^{\#}$ & $42.5 \pm 7.5$ & $41.4 \pm 6.5$ & $42.9 \pm 7.8$ & $43.5 \pm 7.3$ \\
\hline Gender (M/F) & $5 / 24$ & $9 / 10$ & $4 / 17$ & $6 / 18$ \\
\hline BoP (\%)* & $22.0 \pm 3.6^{\mathrm{a}}$ & $39 \pm 16.4^{\mathrm{b}}$ & $26.9 \pm 8.1^{\mathrm{b}}$ & $47.2 \pm 10.8^{\mathrm{c}}$ \\
\hline CAL (mm)* & $2.3 \pm 0.3^{\mathrm{a}}$ & $3.1 \pm 0.5^{\mathrm{b}}$ & $2.4 \pm 0.3^{\mathrm{a}}$ & $3.2 \pm 0.5^{\mathrm{b}}$ \\
\hline
\end{tabular}

BoP - bleeding on probing; CAL - clinical attachment level; NWPH - normal weight/periodontally healthy; NWCP - normal weight/chronic periodontitis; $\mathrm{OPH}$ - obese/periodontally healthy; $\mathrm{OCP}$ - obese/ chronic periodontitis (\#One-Way ANOVA; *Kruskal-Wallis One-Way ANOVA on Ranks/Kruskal-Wallis Multiple-Comparison Z-Value Test [Dunn's Test]; $p<0.05$ ).

Table 2. Serum levels of adipocytokines (mean + standard deviations).

\begin{tabular}{|l|c|c|c|c|}
\hline Parameters & NWPH & NWCP & OPH & OCP \\
\hline Adiponectin (ug/ml)* & $7.2 \pm 5.8^{\mathrm{a}}$ & $10.5 \pm 9.7^{\mathrm{ac}}$ & $17.5 \pm 11.0^{\mathrm{b}}$ & $13.6 \pm 12.6^{\mathrm{bc}}$ \\
\hline Leptin (pg/ml)* & $11.9 \pm 5.2^{\mathrm{ac}}$ & $9.9 \pm 8.9^{\mathrm{a}}$ & $16.4 \pm 5.4^{\mathrm{b}}$ & $13.5 \pm 5.2^{\mathrm{bc}}$ \\
\hline TNF- $\boldsymbol{\alpha}(\mathbf{p g} / \mathbf{m l})^{*}$ & $3.5 \pm 1.3$ & $4.9 \pm 3.1$ & $5.1 \pm 3.9$ & $4.6 \pm 2.5$ \\
\hline
\end{tabular}

BoP - bleeding on probing; CAL - clinical attachment level; NWPH - normal weight/periodontally healthy; NWCP - normal weight/chronic periodontitis; OPH - obese/periodontally healthy; OCP obese/chronic periodontitis; TNF - tumoral necrosis factor Different letters indicate differences between groups (*Kruskal-Wallis One-Way ANOVA on Ranks/Kruskal-Wallis Multiple-Comparison Z-Value Test [Dunn's Test]; $p<0.05)$.

nectin and leptin than the OCP one, but significant higher than NWPH and NWCP $(p<0.05)$. However, leptin levels were significant higher for the OCP group when compared to the NWCP group $(p<0.05)$. For TNF- $\alpha$ le$\mathrm{vel} / \mathrm{s}$, there were no statistically significant differences between groups ( $p>0.05$ ).

The degree of association between obesity and CP was investigated by MLR analyses (Tables 3-6) based on different dependent variables (i.e. diagnosis of obesity, type of obesity [I or II], diagnosis of CP, diagnosis of obesity $+\mathrm{CP}$, and diagnosis of obesity $+\mathrm{CP}$ and presence of site exhibiting PD $>5 \mathrm{~mm}$ ). The first and second MLR models found that obese subjects, with CP or not, present significantly more bleeding sites than normal weight individuals with odds ratio of 0.93 (95\% confidence interval (CI): $-0.88,0.98,-0.01$; Wald $\mathrm{z}$-value $=-2.65 ; \mathrm{p}=0.007)$ and $0.92(95 \%$ confidence interval (CI): $-0.87,-0.98$; Wald $\mathrm{z}$-value $=-2.35 ; p=0.018$ ), respectively. For the third MLR analyses, a significant association between the type of obesity and the suspected independent variables was not found $(p>0.05)$. Regarding the MLR models assessing the influence of obesity on $\mathrm{CP}$, none of them demonstrated statistically significant associations between the suspected independent variables and the tested dependent ones $(p>0.05)$.

\section{Discussion}

It may be difficult to accurately predict periodontitis progression if conditions known to influence the host response are also present. Previous studies have investigated the interaction effects with diabetes mellitus and smoking $(10,19)$. Moreover, it has also been considered the potential impact of periodontal disease on systemic health, such as adequate glycaemic control, cardiovascular and renal diseases $(19,5-8)$. Regarding the influence of $\mathrm{CP}$ on the levels of adipocytokines, the potential association has been considered an important condition in periodontal research. For instance, a recent systematic review found that subjects with periodontal disease have a $30 \%$ greater chance of obesity, as well as higher BMI and CAL loss (18). On the other hand, the same authors did not find prospective observational studies, and thus they considered that it could not be possible to establish causal association (18). The present study failed to sustain these assumptions. Overall, obese subjects with $\mathrm{CP}$ presented more bleeding sites than periodontal healthy ones. Moreover, there was a statistically significant equivalency between normal weight subjects with $\mathrm{CP}$ and obese ones without $\mathrm{CP}$. CAL was similar between healthy groups and between CP groups, but statistically higher when comparing healthy to $\mathrm{CP}$ groups 
Table 3. Multivariable logistic regression analysis estimating the interaction effects between periodontal status with a diagnosis of obesity (overall)

\begin{tabular}{|l|c|c|c|c|cc|}
\hline & OR & SE & $\mathbf{Z}$ & $\boldsymbol{P}>|\mathbf{z}|$ & \multicolumn{2}{|c|}{ 95\% CI } \\
\hline Chronic periodontitis (yes) & 1.85 & 0.68 & 0.90 & 0.368 & 0.48 & 7.08 \\
\hline Gender (male) & 1.66 & 0.53 & 0.95 & 0.341 & 0.58 & 4.72 \\
\hline Age & 0.97 & 0.03 & -0.77 & 0.439 & 0.92 & 1.03 \\
\hline BoP & 0.93 & 0.02 & -2.65 & 0.007 & 0.88 & 0.98 \\
\hline
\end{tabular}

$\mathrm{BoP}$ - bleeding on probing; CI: confidence interval; OR - odds ratio; SE - standard error; $\mathrm{z}$ - Wald z-value.

Table 4. Multivariable logistic regression analysis estimating the interaction effects between periodontal status with a diagnosis of obesity (type I or type II).

\begin{tabular}{|c|c|c|c|c|c|c|}
\hline & & & Obesity type I & & & \\
\hline & OR & SE & $\mathbf{z}$ & $P>|z|$ & \multicolumn{2}{|c|}{$95 \% \mathrm{CI}$} \\
\hline Chronic periodontitis (yes) & 5.03 & 0.96 & 1.67 & 0.093 & 0.76 & 33.19 \\
\hline Gender (male) & 0.76 & 0.60 & -0.43 & 0.664 & 0.23 & 2.52 \\
\hline Age & 0.98 & 0.03 & -0.26 & 0.787 & 0.92 & 1.06 \\
\hline \multirow[t]{3}{*}{ BoP } & 0.92 & 0.03 & -2.35 & 0.018 & 0.87 & 0.98 \\
\hline & & & Obesity type II & & & \\
\hline & OR & SE & $\mathbf{z}$ & $P>|z|$ & \multicolumn{2}{|c|}{$95 \%$ CI } \\
\hline Chronic periodontitis (yes) & 0.61 & 0.66 & -0.72 & 0.469 & 0.16 & 2.28 \\
\hline Gender (male) & 2.35 & 0.59 & 1.43 & 0.151 & 0.73 & 7.61 \\
\hline Age & 0.97 & 0.03 & -0.64 & 0.519 & 0.91 & 1.04 \\
\hline BoP & 0.98 & 0.02 & -0.84 & 0.399 & 0.93 & 1.02 \\
\hline
\end{tabular}

BoP - bleeding on probing;CI: confidence interval; OR - odds ratio; SE - standard error; $\mathrm{z}$ - Wald z-value.

Table 5. Multivariable logistic regression analysis estimating the interaction effects between age, gender, adipocytokines levels and obesity with a diagnosis of chronic periodontitis.

\begin{tabular}{|l|c|c|c|c|cc|}
\hline & OR & SE & $\mathbf{z}$ & $\boldsymbol{P}>|\mathbf{z}|$ & \multicolumn{2}{|c|}{$\mathbf{9 5 \%}$ CI } \\
\hline Age & 1.00 & 0.03 & 0.24 & 0.804 & 0.94 & 1.07 \\
\hline Gender (male) & 0.35 & 0.73 & -1.41 & 0.158 & 0.08 & 1.49 \\
\hline Adiponectin & 1.00 & 0.02 & 0.12 & 0.897 & 0.96 & 1.04 \\
\hline Leptin & 1.01 & 0.05 & 0.22 & 0.819 & 0.91 & 1.12 \\
\hline TNF- $\alpha$ & 0.92 & 0.08 & -0.95 & 0.339 & -0.23 & 0.08 \\
\hline Obesity (yes) & 0.48 & 0.49 & -1.47 & 0.140 & 0.78 & 1.08 \\
\hline
\end{tabular}

$\mathrm{BoP}$ - bleeding on probing; CAL - clinical attachment level; CI: confidence interval; OR - odds ratio; $\mathrm{SE}$ - standard error; $\mathrm{z}$ - Wald z-value.

$(p<0.0001)$. This finding suggests that obese individuals may be at an increased risk of more severe clinical periodontal inflammation (as measured by BoP) even when a healthy periodontal status is present.

Furugen et al. compared the serum levels of different adipocytokines and periodontal status (26) reporting no significant differences in the serum levels of adiponectin and TNF- $\alpha$ between subjects with and without periodontitis. Saito et al. did not find asignificant association between periodontitis and adiponectin in middle-aged. Japanese women (27) found significant lower levels of adiponectin in subjects with periodontitis, as well as 
Table 6. Multivariable logistic regression analysis estimating the interaction effects between age, gender, adipocytokines levels and a diagnosis of obesity + chronic periodontitis.

\begin{tabular}{|c|c|c|c|c|c|c|}
\hline & & & Obesity + CP & & & \\
\hline & OR & SE & $\mathbf{z}$ & $\boldsymbol{P}>|\mathbf{z}|$ & \multicolumn{2}{|c|}{ 95\% CI } \\
\hline Age & 0.98 & 0.03 & -0.52 & 0.599 & 0.91 & 1.05 \\
\hline Gender (male) & 0.60 & 0.78 & -0.63 & 0.525 & 0.13 & 2.83 \\
\hline Adiponectin & 0.98 & 0.02 & -0.73 & 0.464 & 0.94 & 1.02 \\
\hline Leptin & 0.96 & 0.05 & -0.60 & 0.547 & 0.86 & 1.07 \\
\hline \multirow[t]{3}{*}{ TNF- $\alpha$} & 0.97 & 0.08 & -0.26 & 0.790 & 0.82 & 1.15 \\
\hline & & Obesity & $+\mathrm{CP} \quad \mathrm{PD}>$ & $5 \mathrm{~mm}$ & & \\
\hline & OR & SE & $\mathbf{z}$ & $P>|\mathbf{z}|$ & \multicolumn{2}{|c|}{$95 \% \mathrm{CI}$} \\
\hline Age & 1.02 & 0.05 & 0.44 & 0.655 & 0.92 & 1.13 \\
\hline Gender (male) & 0.34 & 1.14 & -0.93 & 0.348 & 0.03 & 3.21 \\
\hline Adiponectin & 0.96 & 0.03 & -1.01 & 0.309 & 0.91 & 1.02 \\
\hline Leptin & 0.98 & 0.08 & -0.19 & 0.842 & 0.83 & 1.15 \\
\hline TNF- $\alpha$ & 1.03 & 0.13 & 0.24 & 0.806 & 0.78 & 1.35 \\
\hline
\end{tabular}

$\mathrm{BoP}$ - bleeding on probing; CAL - clinical attachment level; CI: confidence interval; OR - odds ratio; SE standard error; $\mathrm{z}$ - Wald z-value.

suggested that this anti-inflammatory marker could be used to periodontal assessment (28) did not find a positive relationship between the serum levels of adiponectin, leptin, resistin, interleukin- 6 and TNF- $\alpha$ and CP.

Regarding obesity, to the best of our knowledge, this is the second cross-sectional study designed to assess the relationship between serum levels of adipocytokines and $\mathrm{CP}$ in obese and normal weight subjects. Most of the present outcomes are in line with data from the study performed by Zimmerman et al. (23). On the other hand, several differences between each individual study's outcomes deserve some attention: 1) In this study, all groups presented a similar mean age. Zimmmerman et al. (23) argued that the levels of adipocytokines could be linked to differences in mean age of their groups instead of being linked to $\mathrm{CP}$, as well as that other factors such as ethnicity and gender, might be related to adipose tissue and adipocytokines` levels; 2) Conversely to the previous publications, all the included patients presented similar ethnicity and food intake (Amerindian-Spanish) what may have assisted in recruiting subjects with similar body fat composition and distribution; 3) Inversely to Zimmerman et al. findings (23), the serum levels of adiponectin were similar between obese groups, but higher for OPH when both NW groups were compared $(p=$ $0.0009)$. Moreover, NWCP and OCP groups reported si- milar adiponectin levels supporting the suggestion that periodontal inflammation seems to regulate the level of this anti-inflammatory adipcytokine as well $(23,29)$. It has been shown that adiponectin regulates inflammation by preventing the stimulation of pro-inflammatory cytokines and allowing the discharge of anti-inflammatory mediators (15). However, previous data suggested that the serum levels of adipinectin seem to be lower in subjects with periodontitis when compared to healthy subjects $(26,27)$; 4) Regarding leptin levels, the lowest leptin levels were seen in normal weight subjects with $\mathrm{CP}$ instead of in normal weight subjects without $\mathrm{CP}$, as well as MLR analysis associated obesity to CP (23). It has been shown that leptin leads to an important function in host defense mechanisms because of its role on proinflammatory stimuli (30). In fact, both PH groups and $\mathrm{CP}$ groups presented similar levels (that is, they were not statistically different), but NWPH subjects showed similar outcomes to OCP individuals, and OPH subjects presented the highest levels $(p=0.007)$. These findings suggest that CP may decrease the serum levels of leptin; and 5) it has been suggested that the higher levels of TNF- $\alpha$ reported to OPH in comparison to NWPH could be partially associated to obesity even without the presence of a periodontal diagnosis. The present outcomes do not support this assumption as no significant diffe- 
rences between groups could be detected (neither the Kruskal-Wallis test nor the MLR models). Earlier data positively linked BMI to TNF- $\alpha$ serum levels (31), but methodological discrepancies, such as the criteria used to assess $\mathrm{CP}$ and obesity and the laboratorial tests applied to quantify the serum levels may reflect the differences between samples (23).

In addition, in this cross-sectional study, only a 'snapshot' of the potential link between CP and obesity could be analyzed. Also, the diagnosis of obesity was based on BMI alone as proposed by the World Health Organization (2012); however, it has been argued that a diagnosis based on BMI and waist-hip ratio may provide a better characterization of this condition (23). Besides, gingival crevicular fluid was not collected from sites with PD > $5 \mathrm{~mm}$ to test for differences between serum levels and locally produced inflammatory cytokines. Due to the limited data available, further studies have to be performed to assess the impact of $\mathrm{CP}$ on the serum levels of adipocytokines. Non-significant differences between groups may be associated to type-II statistical error.

\section{Conclusions}

In conclusion and within the limits of this study, it was not possible to establish a positive link between $\mathrm{CP}$ and obesity in terms of differences in the levels of adiponectin, leptin and TNF- $\alpha$ between obese and NW subjects with and without periodontitis. However, obese subjects showed significant more bleeding sites than NW ones. In addition, the serum levels of adipocytokines of periodontally healthy obese subjects were similar to normal weight with CP.

\section{References}

1. Armitage GC. Periodontal diagnoses and classification of periodontal diseases. Periodontol 2000. 2004;34:9-21.

2. Schätzle M, Löe H, Bürgin W, Anerud A, Boysen H, Lang NP. Clinical course of chronic periodontitis. I. Role of gingivitis. J Clin Periodontol. 2003;30:887-901.

3. Schätzle M, Löe H, Lang NP, et al. Clinical course of chronic periodontitis. III. Patterns, variations and risks of attachment loss. J Clin Periodontol. 2003;30:909-18.

4. Chambrone L, Guglielmetti M, Pannuti C, Chambrone L. Evidence grade associating periodontitis to preterm birth and/or low birth weight: I. A systematic review of prospective cohort studies. J Clin Periodontol. 2011;38:795-808

5. Friedewald VE, Kornman KS, Beck JD, et al. The American Journal of Cardiology and Journal of Periodontology Editors' Consensus: periodontitis and atherosclerotic cardiovascular disease. Am J Cardiol. 2009;104:59-68.

6 .Chambrone L, Foz AM, Guglielmetti MR, et al. Periodontitis and chronic kidney disease: a systematic review of the association of diseases and the effect of periodontal treatment on estimated glomerular filtration rate. J Clin Periodontol. 2013;40:443-56.

7. Preshaw PM, Alba AL, Herrera D, et al. Periodontitis and diabetes: a two-way relationship. Diabetologia. 2012;55:21-31.

8. Simpson TC, Needleman I, Wild SH, Moles DR, Mills EJ. Treatment of periodontal disease for glycaemic control in people with diabetes. Aust Dent J. 2010;55:472-4.

9. Tomar SL, Asma S. Smoking-attributable periodontitis in the United States: findings from NHANES III. National Health and Nutrition Examination Survey. J Periodontol. 2000;71:743-51.
10. Chambrone L, Chambrone D, Lima L, Chambrone L. Predictors of tooth loss during long-term periodontal maintenance: a systematic review of observational studies. J Clin Periodontol. 2010;37:675-84.

11. Chambrone L, Preshaw PM, Rosa EF, et al. Effects of smoking cessation on the outcomes of non-surgical periodontal therapy: a systematic review and individual patient data meta-analysis. J Clin Periodontol. 2013;40:607-15.

12. Tesauro M, Mascali A, Franzese O, Cipriani S, Cardillo C, Di Daniele N. Chronic kidney disease, obesity, and hypertension: the role of leptin and adiponectin. Int J Hypertens. 2012;20:943 605.

13. WHO $\mid$ Obesity and overweight [Internet]. WHO2012 [cited 2013 Aug 23];Available from: http://www.who.int/mediacentre/factsheets/ fs $311 / \mathrm{en}$

14. Tesauro M, Mascali A, Franzese O, Cipriani S, Cardillo C, Di Daniele N. Chronic kidney disease, obesity, and hypertension: the role of leptin and adiponectin. Int J Hypertens. 2012;20:943605.

15. Ouchi N, Parker JL, Lugus JJ, Walsh K. Adipokines in inflammation and metabolic disease. Nat Rev Immunol. 2011;11:85-97.

16. Ryo M, Nakamura T, Kihara S, Kumada M, Shibazaki S, Takahashi $\mathrm{M}$. Adiponectin as a biomarker of the metabolic syndrome. Circ J Off J Jpn Circ Soc. 2004;68:975-81.

17. Johnson RB, Serio FG. Leptin within healthy and diseased human gingiva. J Periodontol. 2001;72:1254-7.

18. Chaffee BW, Weston SJ. Association between chronic periodontal disease and obesity: a systematic review and meta-analysis. J Periodontol. 2010;81:1708-24.

19. Suvan J, D'Aiuto F, Moles DR, Petrie A, Donos N. Association between overweight/obesity and periodontitis in adults. A systematic review. Obes Rev. 2011;12:e381-e404.

20. Preshaw PM, Taylor JJ. How has research into cytokine interactions and their role in driving immune responses impacted our understanding of periodontitis? J Clin Periodontol. 2011;38:60-84.

21. Hotamisligil GS, Shargill NS, Spiegelman BM. Adipose expression of tumor necrosis factor-alpha: direct role in obesity-linked insulin resistance. Science. 1993;259:87-91.

22. Karthikeyan BV, Pradeep AR. Gingival crevicular fluid and serum leptin: their relationship to periodontal health and disease. J Clin Periodontol. 2007;34:467-72.

23. Zimmermann GS, Bastos MF, Dias Gonçalves TE, Chambrone L, Duarte PM. Local and Circulating Levels of Adipocytokines in Obese and Normal Weight Individuals With Chronic Periodontitis. J Periodontol. 2012;84:624-33.

24. Von Elm E, Altman DG, Egger M, Pocock SJ, Gøtzsche PC, Vandenbroucke JP. The Strengthening the Reporting of Observational Studies in Epidemiology (STROBE) Statement: guidelines for reporting observational studies. Lancet. 2007;370:1453-7.

25. Armitage GC. Development of a Classification System for Periodontal Diseases and Conditions. Ann Periodontol. 1999;4:1-6.

26. Furugen R, Hayashida $H$, Yamaguchi $N$, et al. The relationship between periodontal condition and serum levels of resistin and adiponectin in elderly Japanese. J Periodontal Res. 2008;43:556-62.

27. Saito T, Yamaguchi N, Shimazaki Y, et al. Serum levels of resistin and adiponectin in women with periodontitis: the Hisayama study. J Dent Res. 2008;87:319-22.

28. Teles FR, Teles RP, Martin L, Socransky SS, Haffajee AD. Relationships Among Interleukin-6, Tumor Necrosis Factor- $\alpha$, Adipokines, Vitamin D, and Chronic Periodontitis. J Periodontol. 2012;83:118391.

29. Nagano Y, Arishiro K, Uene M, et al. A low ratio of high molecular weight adiponectin to total adiponectin associates with periodontal status in middle-aged men. Biomarkers. 2011;16:106-11.

30. Grunfeld C, Zhao C, Fuller J, Pollack A, Moser A, Friedman J, et al. Endotoxin and cytokines induce expression of leptin, the ob gene product, in hamsters. J Clin Invest. 1996;97:2152-7.

31. Genco RJ, Grossi SG, Ho A, Nishimura F, Murayama Y. A proposed model linking inflammation to obesity, diabetes, and periodontal infections. J Periodontol. 2005;76:2075-84. 